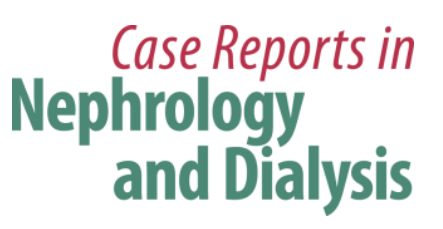

Case Rep Nephrol Dial 2020;10:1-8

DOI: $10.1159 / 000505313$

Published online: January 10, 2020

(c) 2020 The Author(s)

Published by S. Karger AG, Basel

www.karger.com/cnd

This article is licensed under the Creative Commons Attribution-NonCommercial 4.0 International License (CC BY-NC) (http://www.karger.com/Services/OpenAccessLicense). Usage and distribution for commercial purposes requires written permission.

\title{
A Case of Frequently Relapsing Minimal-Change Nephrotic Syndrome with Steroid-Induced Psychiatric Syndrome Treated by Low-Dose, Short-Term Steroid Therapy in Combination with Cyclosporine
}

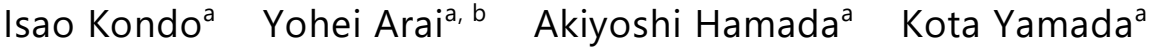 \\ Shingo Shioji ${ }^{a}$ Emi Sakamoto $^{a}$ Daisuke Katagiri $^{a} \quad$ Manami Tada $^{a}$ \\ Fumihiko Hinoshita ${ }^{a}$ \\ aDepartment of Nephrology, National Center for Global Health and Medicine, \\ Tokyo, Japan; bepartment of Nephrology, Graduate School of Medical and Dental \\ Sciences, Tokyo Medical and Dental University, Tokyo, Japan
}

\section{Keywords}

Minimal-change nephrotic syndrome $\cdot$ Steroid-induced psychiatric syndrome $\cdot$ Depression .

Cyclosporine $\cdot$ Prednisolone

\begin{abstract}
Adults with minimal-change nephrotic syndrome (MCNS) generally receive oral prednisolone (PSL) at an initial dosage of $1.0 \mathrm{mg} / \mathrm{kg} / \mathrm{day}$ for a minimum of 4 weeks, with $80 \%$ of patients achieving clinical remission. However, relapses are frequent, necessitating repeated treatment with high-dose PSL. Long-term treatment with high-dose steroids increases the risk of steroid toxicities, such as diabetes mellitus, gastric complications, infections, osteoporosis, and steroid-induced psychiatric syndrome (SIPS), which may compromise the patient's quality of life. Strategies are therefore needed to reduce the dosage and duration of steroid therapy for frequently relapsing MCNS (FRNS). Here, we suggest a new combination therapy of low-dose and
\end{abstract}




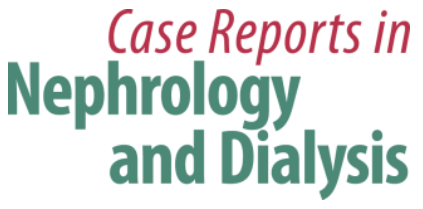

Case Rep Nephrol Dial 2020;10:1-8

DOI: $10.1159 / 000505313$

(c) 2020 The Author(s). Published by S. Karger AG, Basel www.karger.com/cnd

Kondo et al.: Nephrotic Syndrome Treated with Minimal Steroid Therapy and Cyclosporine

short-term steroid with cyclosporine (CsA). We encountered an adult patient who developed recurrence of FRNS with depression arising from SIPS and was treated using low-dose, shortterm PSL combined with CsA. He was successfully treated with PSL at an initial dosage of 0.3 $\mathrm{mg} / \mathrm{kg} /$ day $(20 \mathrm{mg} /$ day) for just 2 weeks combined with CsA, allowing earlier induction of complete remission. We then promptly reduced the dose of PSL to below a physiological dosage ( $5 \mathrm{mg} /$ day) over 3 weeks without relapse after episodes of SIPS and quickly resolved psychiatric symptoms. CSA in combination with PSL can reduce the initial dosage of PSL, shorten the time to remission, and easily maintain clinical remission. This protocol appears clinically useful and potentially applicable as a future treatment strategy for FRNS troubled by SIPS.

(C) 2020 The Author(s)

Published by S. Karger AG, Basel

\section{Introduction}

Prednisolone (PSL) alone at an initial dosage of $1.0 \mathrm{mg} / \mathrm{kg} /$ day is generally administered for a minimum of 4 weeks in adult patients with minimal-change nephrotic syndrome (MCNS), and $80 \%$ of patients with MCNS achieve clinical remission [1]. However, relapses are frequent in a majority of these patients, necessitating repeated courses of treatment with high-dose PSL. Long-term treatment with high-dose steroid increases the risks of various steroid toxicities, including diabetes mellitus, gastric complications, infections, osteoporosis, and steroidinduced psychiatric syndrome (SIPS), which may compromise quality of life [2]. Strategies to reduce the dosage and duration of steroid therapy are thus needed. We suggest a new strategy for frequently relapsing MCNS (FRNS) with SIPS in this case report.

We report herein an adult case of FRNS with depression triggered by SIPS treated by lowdose, short-term steroid therapy in combination with cyclosporine (CsA). This case was successfully treated using PSL at an initial low dosage of $0.3 \mathrm{mg} / \mathrm{kg} /$ day $(20 \mathrm{mg} /$ day) for just 2 weeks in combination with CsA at an initial dosage of $1.5 \mathrm{mg} / \mathrm{kg} /$ day, which induced earlier complete remission. This initial dose was promptly reduced to below physiological dosage ( $5 \mathrm{mg}$ /day) over 3 weeks without relapse after episodes of SIPS, and psychiatric symptoms quickly resolved. We suggest that this treatment protocol is valid and can be applied as a future treatment strategy for FRNS presenting with SIPS.

\section{Case Report}

A 51-year-old man was admitted to our hospital in mid-April with a history of edema in the lower extremities since the beginning of April. Medical history included FRNS at 15 years of age that had been treated using PSL at an initial dosage of $1.0 \mathrm{mg} / \mathrm{kg} /$ day at the time of relapse. Steroid therapy had finished by 42 years of age, after which time complete remission was maintained. Since he had developed depression as a part of SIPS at 16 years of age, he had been regularly seeing a psychiatrist and had maintained control of psychiatric symptoms under a stable condition using fluvoxamine maleate acid at $300 \mathrm{mg} /$ day.

On admission, body weight was $68.1 \mathrm{~kg}$, representing a gain of about $10 \mathrm{~kg}$ to a higher than usual weight over the preceding 2 weeks. Blood pressure was also high, at 159/103 mm Hg. Major laboratory examinations on admission showed: white blood cells, 7,340/ $\mu \mathrm{L}$; blood hemoglobin, $13.7 \mathrm{~g} / \mathrm{dL}$; platelet count, $36.1 \times 10^{4} / \mu \mathrm{L}$; partial thromboplastin time, $10.3 \mathrm{~s}$; activated partial thromboplastin time, $23.0 \mathrm{~s}$; D-dimer, $3.4 \mu \mathrm{g} / \mathrm{mL}$; total protein, $4.3 \mathrm{~g} / \mathrm{dL}$; serum albumin, $1.5 \mathrm{~g} / \mathrm{dL}$; lactate dehydrogenase, $240 \mathrm{U} / \mathrm{L}$; aspartate aminotransferase, $17 \mathrm{U} / \mathrm{L}$; 


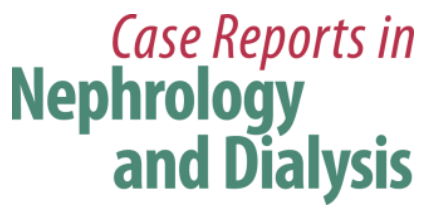

alanine aminotransferase, $7 \mathrm{U} / \mathrm{L}$; low-density lipoprotein cholesterol, $326 \mathrm{mg} / \mathrm{dL}$; triglyceride, $278 \mathrm{mg} / \mathrm{dL}$; blood urea nitrogen, $12.9 \mathrm{mg} / \mathrm{dL}$; serum creatinine, $0.85 \mathrm{mg} / \mathrm{dL}$; sodium, 142 $\mathrm{mEq} / \mathrm{L}$; potassium, $4.2 \mathrm{mEq} / \mathrm{L}$; chloride, $104 \mathrm{mEq} / \mathrm{L}$; C-reactive protein, $0.03 \mathrm{mg} / \mathrm{dL}$; glycated hemoglobin (HbA1c), 5.6\%; C3, $167 \mathrm{mg} / \mathrm{dL} ; \mathrm{C} 4,40.3 \mathrm{mg} / \mathrm{dL}$; hemolytic complement activity (CH50), 58 mg/dL; immunoglobulin (Ig)G, 949 mg/dL; IgA, 398 mg/dL; IgM, 52 mg/dL; antinuclear antibody, (-); and antineutrophil cytoplasmic antibodies, (-). Urinalysis revealed: proteinuria, $7.8 \mathrm{~g}$ /day; glycosuria, (-); occult blood, (-); and urinary protein selectivity, 0.20 . Immunoelectrophoretic study showed no myeloma protein. Chest X-ray disclosed mild bilateral pleural effusion.

These findings suggested relapse of MCNS because of the high selective glomerular proteinuria, hypoalbuminemia, hyperlipidemia, and peripheral edema. We therefore performed percutaneous renal biopsy and obtained the following findings. Light microscopy revealed 29 glomeruli, none of which were globally or segmentally sclerotic. Glomeruli were nearly normal but showed up to mild mesangial proliferation (Fig. 1a). Immunofluorescent staining showed normal findings. However, electron microscopy showed diffuse effacement of the foot processes (Fig. 1b). Finally, we reached a definitive diagnosis of recurrent FRNS.

We started immunosuppressive therapy with PSL at an initial dosage of $0.3 \mathrm{mg} / \mathrm{kg} /$ day $(20 \mathrm{mg} /$ day) in combination with CsA at an initial dosage of $1.5 \mathrm{mg} / \mathrm{kg} /$ day (Fig. 2). Standard immunosuppressive therapy for recurrent FRNS is PSL alone at an initial dosage of 1.0 $\mathrm{mg} / \mathrm{kg} /$ day for a minimum of 4 weeks, but the history of depression as a part of SIPS necessitated a reduction in the dosage and duration of steroid therapy. On the other hand, CsA requires cautious use to avoid CsA-induced nephrotoxicity. To prevent CsA-induced nephropathy, maintaining the appropriate blood CsA concentration within a narrow therapeutic window appears essential. Monitoring of CsA was thus performed using concentrations at 2-h post-dose, with a target window of $600-900 \mathrm{ng} / \mathrm{mL}$ [3]. Complete remission of FRNS was achieved by 2 weeks after starting treatment, but SIPS recurred the day after remission. We thus promptly reduced the dosage of PSL (weekly reduction by $2.5-5 \mathrm{mg} / \mathrm{day}$ ), and SIPS quickly showed improvements. The dosage of PSL was $5 \mathrm{mg} /$ day as a maintenance dose at 5 weeks after the start of treatment, and complete remission was further maintained without relapse.

\section{Discussion}

The present case of FRNS was successfully treated using low-dose, short-term steroid therapy in combination with CsA, which can induce earlier complete remission and allow prompt reductions in the dosage of PSL without relapse after episodes of SIPS. The benefits of administering CsA in combination with PSL in the initial phase of therapy are based on 3 points. First, CsA can reduce the initial dosage of PSL needed to induce complete remission of FRNS. Second, CsA can shorten the time to remission. Third, CsA can help to maintain remission and promptly reduce the dosage of PSL after episodes of SIPS. These advantages can ease the symptoms of SIPS and allow rapid resolution.

Psychiatric adverse effects are common during steroid therapy. Severe SIPS occurs in $5.7 \%$ of patients, while mild to moderate symptoms occur in $27.6 \%$ [4]. The most common psychiatric disorders are depression (35\%), mania (31\%), psychosis (14\%), delirium (13\%), and mixed states (6\%). Most reactions occur early in the course of steroid therapy (i.e., within 1-2 weeks) [5]. The steroid dosage is the most important risk factor for the development of psychiatric symptoms and a strong dose-response relationship has been demonstrated in 


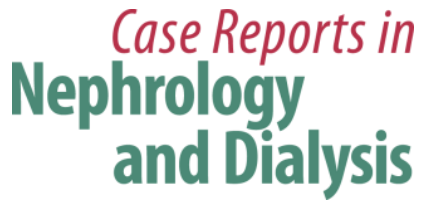

Case Rep Nephrol Dial 2020;10:1-8

DOI: $10.1159 / 000505313$

(c) 2020 The Author(s). Published by S. Karger AG, Basel www.karger.com/cnd

Kondo et al.: Nephrotic Syndrome Treated with Minimal Steroid Therapy and Cyclosporine

various studies [6]. High-dose PSL ( $>40 \mathrm{mg} /$ day) shows a particular increase in the risk of SIPS, but even relatively low doses of PSL (10-20 mg/day) are considered likely to trigger mild psychiatric symptoms. Treatment of SIPS involves discontinuation of the steroids, if possible, or at least an attempt to taper the dose of PSL to below a physiological dosage of 7.5 $\mathrm{mg}$ /day as rapidly as safely possible [7]. Psychiatric disturbances resolve slowly after reducing the dosage of PSL within a few weeks [8]. We thus have to develop treatment strategies to avoid high-dose or long-term steroid therapies and to promptly reduce dosages of PSL after episodes of SIPS.

Therapeutic regimens combining PSL with CsA have more recently been examined in MCNS $[9,10]$. A previous study showed that steroid therapy (initial dosage, $0.8 \mathrm{mg} / \mathrm{kg} /$ day) in combination with CsA to adults with the first relapse of MCNS significantly reduces the time to remission and allows the PSL dose to be reduced more rapidly than with PSL monotherapy (initial dosage, $1.0 \mathrm{mg} / \mathrm{kg} /$ day) [9]. Another report showed that the complete remission rate from CsA monotherapy for adult-onset MCNS was inferior to that from PSL monotherapy (initial dosage, $1.0 \mathrm{mg} / \mathrm{kg} /$ day), but no significant difference in the frequency of relapses was seen [10]. These studies suggest that CsA combination therapy with PSL can reduce the initial dosage of PSL to induce complete remission, shorten time to remission, and easily maintain clinical remission. This combination therapy of CsA with PSL was suggested to be superior to CsA monotherapy in cases with FRNS. We therefore think that combining CsA with PSL is reasonably effective in treating FRNS troubled by SIPS.

CsA should be administered for a year, then slowly tapered. During CsA tapering, 25\% of patients with FRNS can be progressively weaned from CsA and enjoy stable remission [11]. However, over $60 \%$ of patients with FRNS who respond to CsA reportedly relapse after CsA discontinuation [11]. Thus, if a relapse occurs, we need to think about other treatment strategies. In the present case, increasing the dosage of PSL may have caused SIPS recurrence. If this regimen had not proven effective, a method that did not involve increasing the dosage of PSL would have been required.

In recent years, rituximab (RTX) has generally been used in the treatment of patients with PSL- and/or CsA-dependent FRNS, particularly for children [12]. RTX may therefore be considered as the first-line immunosuppressive drug for children. Furthermore, a previous report demonstrated that additional administration of RTX can allow a reduction in the dosage of PSL needed for induction and maintenance of clinical remission in adults [13]. However, no randomized controlled trials have directly compared RTX to CsA in adults. Moreover, progressive multifocal leukoencephalopathy, one of the life-threatening adverse effects of RTX, undeniably occurs more frequently with increasing age [14]. We therefore suggest RTX as a secondline therapy for adults with FRNS.

While no previous randomized trials have established low-dose steroid therapy $<0.5$ $\mathrm{mg} / \mathrm{kg} /$ day for FRNS, a retrospective cohort study demonstrated that high-dose PSL $\quad>20$ $\mathrm{mg} /$ day) is not associated with favorable outcomes after first relapse as compared to a lower dose (10-20 mg/day) [15]. Actually, this observational study had several limitations. First, a number of patients with sub-nephrotic proteinuria were included as relapse cases. Second, no regular protocol was defined for PSL administration with or without other immunosuppressive drugs for relapsed patients. However, the fact remains that a high initial dose of PSL is likely to be dispensable for FRNS. Well-designed randomized, controlled trials are required in the future, and we hope that the therapeutic protocol described in the present case report will see application in future work. 


\section{Case Reports in Nephrology and Dialysis}

\section{Conclusion}

We successfully treated a case of recurrent FRNS with depression as SIPS triggered by PSL at an initial dosage of $0.3 \mathrm{mg} / \mathrm{kg} /$ day ( $20 \mathrm{mg} /$ day) for just 2 weeks in combination with CsA at an initial dosage of $1.5 \mathrm{mg} / \mathrm{kg} /$ day, which could induce earlier complete remission, allow prompt reduction in the dose of PSL to below a physiological dosage (5 mg/day) over 3 weeks without relapse after episodes of SIPS, and quickly resolve psychiatric symptoms. We suggest that this treatment protocol is valid and can be applied in a future treatment strategy for FRNS complicated by SIPS.

\section{Statement of Ethics}

All treatments and examinations followed the guidance of the Declaration of Helsinki. Informed consent for treatment and renal biopsy was obtained from the patient.

\section{Disclosure Statement}

The authors have no conflicts of interest to declare.

\section{Funding Sources}

This project was not supported by any grant or funding agencies.

\section{Author Contributions}

Isao Kondo contributed to acquisition of data and writing of the manuscript. Yohei Arai and Fumihiko Hinoshita provided supervision and mentorship. All the authors approved the final manuscript.

\section{References}

1 Korbet SM, Schwartz MM, Lewis EJ. Minimal-change glomerulopathy of adulthood. Am J Nephrol. 1988;8(4):291-7.

2 Czock D, Keller F, Rasche FM, Häussler U. Pharmacokinetics and pharmacodynamics of systemically administered glucocorticoids. Clin Pharmacokinet. 2005;44(1):61-98.

3 Naito M, Takei T, Eguchi A, Uchida K, Tsuchiya K, Nitta K. Monitoring of blood cyclosporine concentration in steroid-resistant nephrotic syndrome. Intern Med. 2008;47(18):1567-72.

4 Lewis DA, Smith RE. Steroid-induced psychiatric syndromes. A report of 14 cases and a review of the literature. J Affect Disord. 1983 Nov;5(4):319-32.

5 Hall RC, Popkin MK, Stickney SK, Gardner ER. Presentation of the steroid psychoses. J Nerv Ment Dis. 1979 Apr;167(4):229-36.

6 Boston Collaborative Drug Surveillance Program. Acute adverse reactions to prednisone in relation to dosage. Clin Pharmacol Ther. 1972 Sep-Oct;13(5):694-8.

7 Varney NR, Alexander B, MacIndoe JH. Reversible steroid dementia in patients without steroid psychosis. Am J Psychiatry. 1984 Mar;141(3):369-72.

8 Brown ES, Suppes T. Mood symptoms during corticosteroid therapy: a review. Harv Rev Psychiatry. 1998 Jan-Feb;5(5):239-46. 
9 Eguchi A, Takei T, Yoshida T, Tsuchiya K, Nitta K. Combined cyclosporine and prednisolone therapy in adult patients with the first relapse of minimal-change nephrotic syndrome. Nephrol Dial Transplant. 2010 Jan;25(1):124-9.

10 Matsumoto H, Nakao T, Okada T, Nagaoka Y, Takeguchi F, Tomaru R, et al. Favorable outcome of low-dose cyclosporine after pulse methylprednisolone in Japanese adult minimal-change nephrotic syndrome. Intern Med. 2004 Aug;43(8):668-73.

11 Meyrier A. Treatment of idiopathic nephrosis by immunophillin modulation. Nephrol Dial Transplant. 2003 Aug;18(90006 Suppl 6):vi79-86.

12 Basu B, Sander A, Roy B, Preussler S, Barua S, Mahapatra TK, et al. Efficacy of Rituximab vs Tacrolimus in Pediatric Corticosteroid-Dependent Nephrotic Syndrome: A Randomized Clinical Trial. JAMA Pediatr. 2018 Aug;172(8):757-64.

13 Ruggenenti $\mathrm{P}$, Ruggiero B, Cravedi P, Vivarelli M, Massella L, Marasà M, et al.; Rituximab in Nephrotic Syndrome of Steroid-Dependent or Frequently Relapsing Minimal Change Disease Or Focal Segmental Glomerulosclerosis (NEMO) Study Group. Rituximab in steroid-dependent or frequently relapsing idiopathic nephrotic syndrome. J Am Soc Nephrol. 2014 Apr;25(4):850-63.

14 Bennett CL. Pharmacovigilance and PML in the oncology setting. Cleve Clin J Med. 2011 Nov;78 Suppl 2:S137.

15 Ozeki T, Ando M, Yamaguchi M, Katsuno T, Kato S, Yasuda Y, et al. Treatment patterns and steroid dose for adult minimal change disease relapses: A retrospective cohort study. PLoS One. 2018 Jun;13(6):e0199228. 


\section{Case Reports in Nephrology and Dialysis}

Case Rep Nephrol Dial 2020;10:1-8

DOI: $10.1159 / 000505313$

(C) www.karger.com/cnd

Kondo et al.: Nephrotic Syndrome Treated with Minimal Steroid Therapy and Cyclosporine
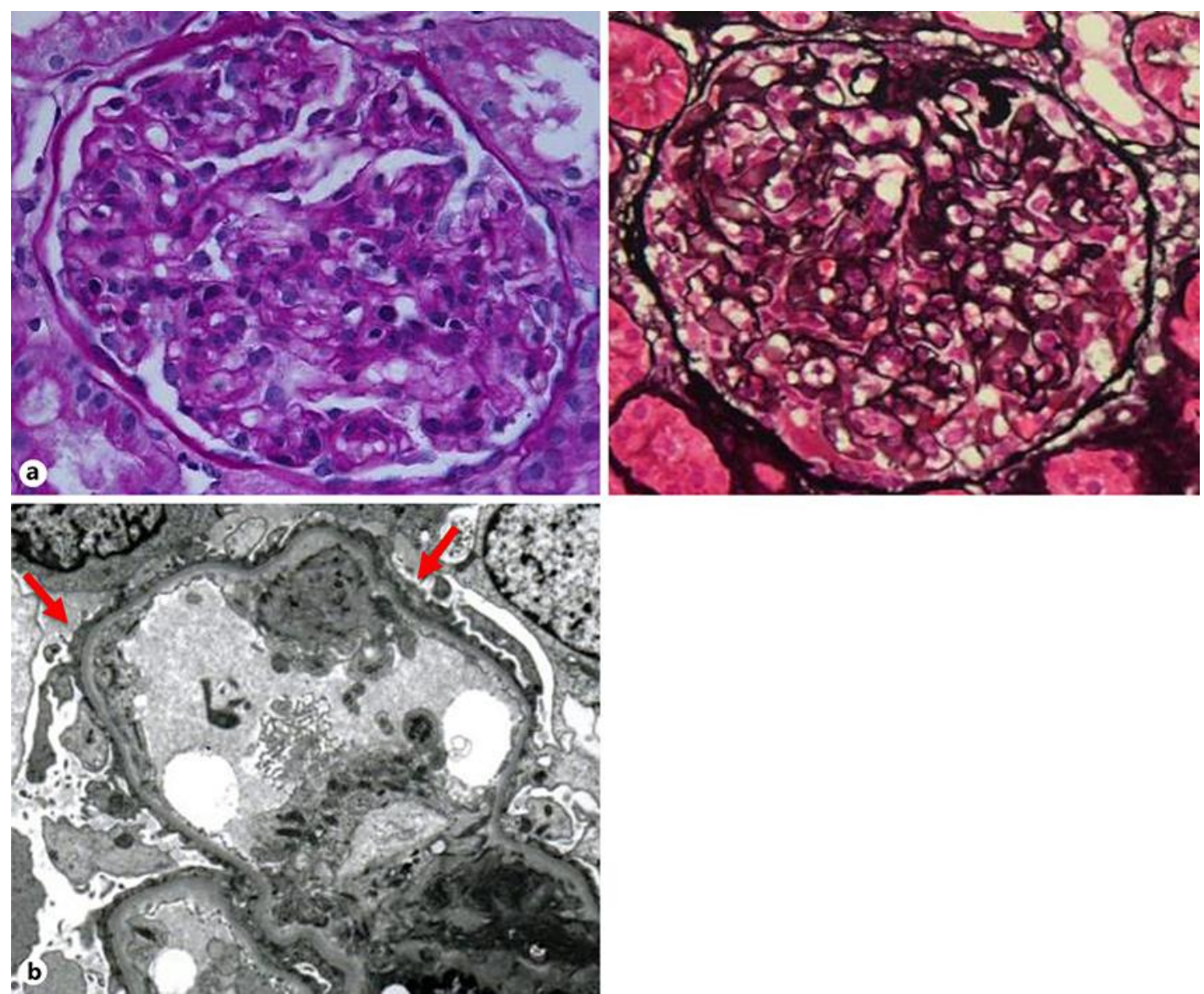

Fig. 1. Pathological findings of renal biopsy. a Light microscopy reveals 29 glomeruli, none of which are globally or segmentally sclerotic. Glomeruli show up to mild mesangial proliferation. Left: Periodic acidSchiff stain (original magnification, $\times 400$ ). Right: Periodic acid-methenamine-silver stain (original magnification, $\times 400)$. b Electron microscopy shows diffuse effacement of the foot processes $(\uparrow$, arrowed line), with no electron-dense material in the mesangium (original magnification, $\times 3,000$ ). 


\section{Clinical Course}
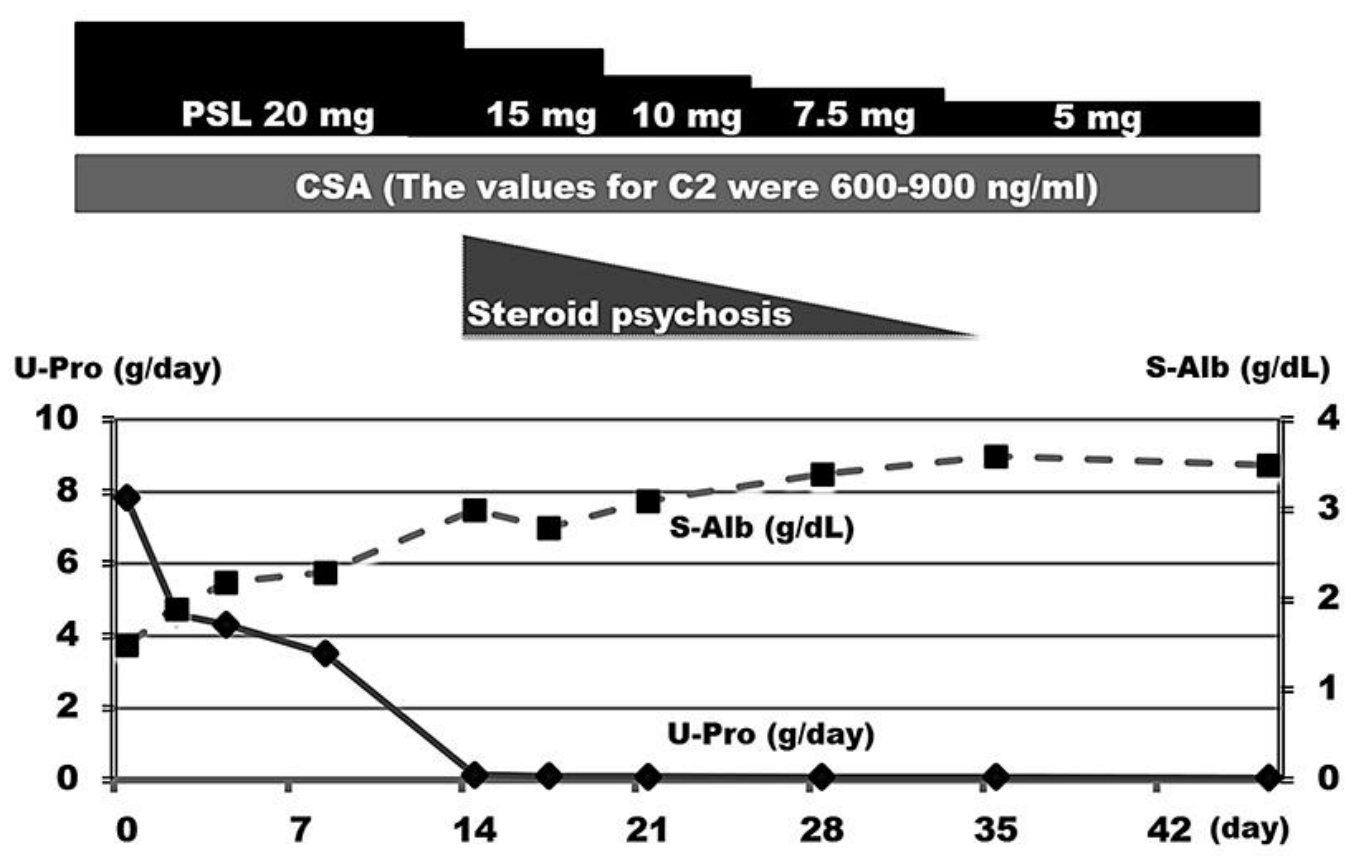

Fig. 2. Clinical course. S-Alb: serum albumin (g/dL, - - -). U-Pro: urine protein (g/day, - - -). Monitoring of cyclosporine was with 2-h post-dose (C2) levels. Values for C2 were $600-900 \mathrm{ng} / \mathrm{mL}$. 